\title{
Neutrophilic leukocytosis in advanced stage polycythemia vera: hematopathologic features and prognostic implications
}

Leonardo Boiocchi ${ }^{1,2}$, Umberto Gianelli ${ }^{2}$, Alessandra Iurlo ${ }^{3}$, Falko Fend ${ }^{4}$, Irina Bonzheim ${ }^{4}$, Daniele Cattaneo ${ }^{3}$, Daniel M Knowles ${ }^{1}$ and Attilio Orazi ${ }^{1}$

${ }^{1}$ Department of Pathology and Laboratory Medicine, Weill Cornell Medical College/New York Presbyterian Hospital, New York, NY, USA; ${ }^{2}$ Hematopathology Service, Division of Pathology, Department of

Pathophysiology and Transplantation, University of Milan and IRCCS Ca' Granda - Ospedale Maggiore Policlinico Foundation, Milan, Italy; ${ }^{3}$ Oncohematology Unit of the Elderly, Division of Oncohematology, IRCCS Ca' Granda - Ospedale Maggiore Policlinico Foundation, Milan, Italy and ${ }^{4}$ Department of Pathology, University of Tubingen, Tubingen, Germany

Polycythemia vera in $\mathbf{2 0 - 3 0 \% ~ o f ~ c a s e s ~ p r o g r e s s e s ~ t o w a r d s ~ p o s t - p o l y c y t h e m i c ~ m y e l o f i b r o s i s , ~ a n ~ a d v a n c e d ~ p h a s e ~}$ characterized by decreased red blood cells counts and increasing splenomegaly with extramedullary hematopoiesis. There is evidence that the presence of neutrophilic leukocytosis at polycythemia vera disease outset is associated with an increased risk of recurrent thrombosis. However, its clinical significance when developing later in the course of the disease is not well defined. Over a period of 8 years we identified from the files of two reference centers 10 patients $(7 \mathrm{M} / 3 \mathrm{~F}$, median age: 68 years) who developed persistent absolute leukocytosis $\geq 13 \times 10^{9} / \mathrm{l}$ (median: $25.1 \times 10^{9} / \mathrm{l}$; range: $16.1-89.7 \times 10^{9} / \mathrm{l}$ ) at or around the time of diagnosis of post-polycythemic myelofibrosis (median interval from diagnosis:0 months; range: $-6 / 31$ ) and persisted for a median period of 13 months. Peripheral blood smears showed numerous neutrophils without dysplastic features and, in four, $\geq 10 \%$ immature myeloid precursors. In five cases, corresponding marrow specimens obtained at or immediately after the onset of leukocytosis showed a markedly increased myeloid:erythroid ratio due to granulocytic proliferation. No change in JAK2 and BCR-ABL1 status or cytogenetic evolution was associated with the development of leukocytosis. The mutational status of CSF3R, SETBP1, and SRSF2, genes associated with other chronic myeloid neoplasms where neutrophilic leukocytosis occurs, was investigated but all cases showed wild-type only alleles. Four patients died after developing leukocytosis and one experienced worsening disease. Compared with a control group of post-polycythemic myelofibrosis patients $(n=23)$ who never developed persistent leukocytosis, patients with leukocytosis showed higher white blood cells counts and a shorter overall survival. This is the first study describing the development of significant neutrophilic leukocytosis during advanced stages of polycythemia vera; it includes comprehensive hematologic, marrow morphological, molecular, and clinical data. Our findings suggest that persistent leukocytosis occurring at or around the time of progression to post-polycythemic myelofibrosis is associated with an overall more aggressive course of the disease.

Modern Pathology (2015) 28, 1448-1457; doi:10.1038/modpathol.2015.100; published online 4 September 2015

Polycythemia vera, one of the classic Philadelphia chromosome/BCR-ABL1 negative myeloproliferative neoplasms, is a relatively indolent disease with a

Correspondence: Dr A Orazi, MD, FRCPath (Engl.), Department of Pathology and Laboratory Medicine, Weill Cornell Medical College/New York Presbyterian Hospital, Room ST-707, 525 East 68th Street, New York, NY 10065, USA.

E-mail: ato9002@med.cornell.edu

Part of this work was presented as at the 103rd Annual Meeting of the United States and Canadian Academy of Pathology in San Diego, California, 1-8 March 2014.

Received 15 June 2015; accepted 6 August 2015; published online 4 September 2015 median survival time from diagnosis commonly exceeding 10 years with current therapies. Polycythemia vera is associated with an increased cardiovascular risk with at least $20 \%$ of deaths caused by thromboembolic or hemorrhagic complications. It consists of a polycythemic phase characterized by a mild to overt excess of red blood cells with increased hemoglobin and hematocrit values often associated with reduced erythropoietin levels ${ }^{1-4}$ and a distinctively panmyelotic hypercellular bone marrow. This polycythemic phase may be followed by myelofibrotic phase, which occurs after $10-15$ years from the onset in $20-30 \%$ of cases. This 
late disease stage, which is usually referred to as post-polycythemic myelofibrosis, is characterized by moderate to severe bone marrow fibrosis with collagen deposition, decreasing blood counts and worsening splenomegaly due to the development of extramedullary hematopoiesis., ${ }^{1,5,6}$ Postpolycythemic myelofibrosis is most likely the result of progressive accumulation of multiple genetic abnormalities occurring during the course of polycythemia vera. Patients with post-polycythemic myelofibrosis display a significantly higher frequency of complex karyotypes often with multiple clones when compared with patients in polycythemic stage, as described in a recent study from our group. ${ }^{6}$ Progression toward acute myeloid leukemia is observed in 10-20\% of all polycythemia vera cases and occurs more often during the post-polycythemic myelofibrotic stage. This blast phase clinically manifests as rapidly progressing marrow failure with presence of $\geq 20 \%$ blasts in bone marrow or peripheral blood. In some patients clinical progression toward acute leukemia is abrupt, whereas in others transformation can be preceded by an 'accelerated phase' characterized by $\geq 10 \%$ myeloblasts in bone marrow and/or peripheral blood, with or without concurrent myelodysplastic syndrome-like morphologic changes.

According to current diagnostic criteria, myelofibrotic progression and accelerated/leukemic blast phase represent the only forms of disease progression recognized in polycythemia vera. However, in patients with Philadelphia chromosome negative myeloproliferative neoplasms, especially in those with a longstanding disease, additional less common and yet not well-recognized manifestations of disease progression may occur. In this regards, it has been recently reported by our group ${ }^{7}$ that a small subset of patients with primary myelofibrosis can develop during their disease a sustained absolute monocytosis in peripheral blood associated with morphologic changes in marrow resembling those typically seen in patients with chronic myelomonocytic leukemia. ${ }^{8,9}$ This occurrence, which is associated with poor prognosis, was considered to be evidence of disease progression. ${ }^{7}$

In polycythemia vera elevated leukocyte counts at disease onset or early during the polycythemic phase of the disease have been identified recently as an independent unfavorable prognostic factor in a few series. ${ }^{10,11}$ The mechanisms determining the negative impact of leukocytosis on survival have not been defined yet, although a few studies have shown that neutrophilia can favor platelet activation and increase the risk of thrombotic events. ${ }^{12,13}$

In this study we describe the clinical and morphologic features of patients with polycythemia vera who developed persistent and significant neutrophilic leukocytosis for the first time at or around the time of their progression to postpolycythemic myelofibrosis. In these patients no increase in the number of blasts, other evidence of accelerated phase/blast phase or increase in the percentage of monocytes were observed. Our study provides for the first time comprehensive clinical, laboratory, cytogenetic, and molecular data seen in these patients, in addition to their detailed peripheral blood and bone marrow findings and a comparison with a control group of postpolycythemic myelofibrosis patients who never developed persistent leukocytosis.

\section{Materials and methods}

\section{Case Selection}

We reviewed the cases of polycythemia vera in the files of the Department of Pathology and Laboratory Medicine of Weill Cornell Medical College/New York Presbyterian Hospital in New York City and of the Department of Pathology of IRCCS Ca' GrandaOspedale Maggiore Policlinico Foundation in Milan to identify polycythemia vera patients progressed to post-polycythemic myelofibrosis. Among 280 patients diagnosed with polycythemia vera between 2006 and 2013, 55 cases of post-polycythemic myelofibrosis were identified. In 10 of the patients, the clinical records were incomplete and those cases were excluded. Review of the clinical charts identified 10 patients who developed for the first time significant leukocytosis, defined as a white blood cells (WBC) count $\geq 13 \times 10^{9} / 1$ persistent for at least 3 months within 6 months before the histological diagnosis of post-polycythemic myelofibrosis or at any time after the diagnosis of post-polycythemic myelofibrosis, and for whom CBC results and peripheral blood smear and bone marrow examination were available. None of the selected cases had shown leukocytosis at the onset of polycythemia vera or earlier during the polycythemic phase of the disease. Careful review of the clinical data of these post-polycythemic myelofibrosis with leukocytosis patients allowed the exclusion of any eventual clinical condition or treatment known to be possibly associated with reactive leukocytosis and neutrophilia. Of the remaining 35 post-polycythemic myelofibrosis cases that did not develop persistent significant leukocytosis, 23 for whom CBC results and peripheral blood smear and bone marrow examination were available were selected as controls. Their clinical and morphological features and survival were compared with those of postpolycythemic myelofibrosis cases with leukocytosis and they served as a control group. None of the selected control group patients had shown leukocytosis at the onset of polycythemia vera or earlier during the polycythemic phase of the disease. The remaining 12 post-polycythemic myelofibrosis cases, which included mostly consultation cases, were not studied owing to the suboptimal quality of their bone marrow specimens and/or lack of peripheral blood smear. 
The design of the study was approved by the Institutional Review Boards of Weill Cornell Medical College, IRCCS Ca' Granda-Ospedale Maggiore Policlinico Foundation and University of Tubingen.

\section{Morphologic Analysis}

Histologic sections of the post-polycythemic myelofibrosis bone marrow biopsy, bone marrow aspirate and peripheral blood smears were reviewed by AO, LB, and UG. When possible, the initial bone marrow biopsy diagnostic for polycythemia vera was also reviewed; in cases in which the initial bone marrow biopsy was not available, eg, cases with an outside diagnosis, the original pathology report and patients' clinical history was carefully reviewed to make sure the clinical diagnosis of polycythemia vera was well established at the time of disease presentation and during the ensuing follow-up, according to the criteria published by the WHO in its 2008 monograph. ${ }^{1}$

Bone marrow biopsies from Weill Cornell Medical College were fixed in Bouin's solution, decalcified in a nitric acid solution and embedded in paraffin; biopsies from Ospedale Maggiore Policlinico were fixed in neutral buffered formalin, decalcified in ethylenediaminetetraacetic acid and embedded in paraffin. Sections from each case were stained with hematoxylin and eosin and Giemsa staining for morphologic examination and Gomori's silver impregnation and Masson's trichrome staining to evaluate fibrosis. ${ }^{2}$ The degree of bone marrow fibrosis was assessed by using the European Consensus Grading System. ${ }^{14}$ Bone marrow aspirates and peripheral blood smears corresponding to the bone marrow biopsies were stained with WrightGiemsa staining. All cases were immunostained with monoclonal antibodies for myeloperoxidase, glycophorin C, CD42b, CD34, and CD117 using external negative and positive controls.

\section{Cytogenetic and Molecular Analysis}

Results of conventional cytogenetic analysis and of Florescence in situ hybridization were reviewed when available. Molecular analysis for the determination of the JAK2 phenotypes was performed on peripheral blood samples obtained close to the time of bone marrow examination. Mutations of exon 14 and the hotspot region (amino acids 739-795) of exon 17 of $C S F 3 R$, of the hotspot region of exon 4 (amino acids 830-892) of SETBP1 and of the hotspot region around P95 of SRSF2 were investigated using DNA extracted from paraffin sections. ${ }^{15,16}$

\section{Follow-up}

Follow-up data were available for all patients. Overall survival was calculated as the interval from the first diagnosis of polycythemia vera to the last follow-up visit; survival times from the diagnosis of post-polycythemic myelofibrosis, from the onset of leukocytosis to the last contact with patient or death and also total duration of leukocytosis were calculated. In case of death, the specific cause was recorded.

\section{Statistical Analysis}

Statistical analysis were performed with the SPSS statistics 17 software (SPSS, Chicago, IL, USA). For all statistical tests a $P$-value $<0.05$ was considered significant. All tests were two-sided. T-test was performed to evaluate differences in continuous variables as, eg, hemoglobin, white cells, neutrophils, and platelets counts, absolute cellularity and myeloid:erythroid ratio. Pearson Chi-squared test was used to evaluate proportions. Survivals in the neutrophilic post-polycythemic myelofibrosis patients and in the control group were described by Kaplan-Meier curves and differences evaluated with log-rank test.

\section{Results}

\section{Cases}

The cohort of post-polycythemic myelofibrosis patients who developed leukocytosis was composed of seven men and three women with a median age of 68 years. In three patients (30\%) leukocytosis was present at time of post-polycythemic myelofibrosis diagnosis, in three $(30 \%)$ it developed within 6 months before the diagnosis, and in the remaining four $(40 \%)$ after the diagnosis. The median time from diagnosis to development of leukocytosis was 0 months. Median duration of leukocytosis was 13 months (see Table 1). The median absolute number of leukocytes was $25.1 \times 10^{9} / 1$ with neutrophils accounting for $\geq 75 \%$ of total WBC in 9 out of 10 cases (Table 2). Increased WBC and absolute neutrophilia and were variably associated with hemoglobin levels and platelets counts. None of the patients had undergone splenectomy at time of diagnosis of postpolycythemic myelofibrosis but most (7 out of 10) showed splenomegaly. Data about treatment during the polycythemic phase was available for nine cases: all patients had received phlebotomies, hydroxyurea, anagrelide, and aspirin in various combinations; patient 6 had also received interferon and melphalan; during the myelofibrotic phase, phlebotomies were suspended in all patients and most of them continued receiving aspirin variously combined with hydroxyurea and ruxolitinib; patient 2 was treated exclusively with interferon (Table 3).

The clinical features of the 23 cases of the control group are summarized in Table 4. Age, sex distributions, overall disease length, and follow-up times both before and after the diagnosis of postpolycythemic myelofibrosis were similar to those of 
Table 1 Age, sex, total duration of leukocytosis, interval from post-polycythemic myelofibrosis diagnosis to onset of leukocytosis, followup time after the development of leukocytosis, and overall survival from the diagnosis of polycythemia vera in patients with postpolycythemic myelofibrosis and leukocytosis

\begin{tabular}{|c|c|c|c|c|c|c|}
\hline Case & Age & Sex & $\begin{array}{l}\text { Total duration of } \\
\text { leukocytosis (months) }\end{array}$ & $\begin{array}{c}\text { Post-PV MF/onset of } \\
\text { leukocytosis interval (months) }\end{array}$ & $\begin{array}{l}\text { Survival after onset of } \\
\text { leukocytosis (months) }\end{array}$ & OS (months) \\
\hline 1 & 53 & $\mathrm{~F}$ & 3 & 0 & 13 & 120 \\
\hline 2 & 74 & $\mathrm{~F}$ & 14 & 6 & 16 & 279 \\
\hline 3 & 70 & $\mathrm{M}$ & 11 & 0 & 27 & 72 \\
\hline 4 & 67 & $\mathrm{M}$ & 14 & 5 & 15 & 54 \\
\hline 5 & 75 & $\mathrm{M}$ & 14 & 1 & 45 & 190 \\
\hline 6 & 71 & $\mathrm{~F}$ & 4 & 4 & 88 & 300 \\
\hline 7 & 59 & $\mathrm{M}$ & 15 & 0 & 35 & 253 \\
\hline 8 & 69 & $\mathrm{M}$ & 3 & 26 & 40 & 170 \\
\hline 9 & 68 & M & 12 & 31 & 32 & 210 \\
\hline 10 & 50 & $\mathrm{M}$ & 16 & 23 & 16 & 171 \\
\hline Range & $50 / 75$ & - & $3 / 16$ & $6 / 31$ & $13 / 88$ & $54 / 300$ \\
\hline Mean & 66 & - & 11 & 7 & 33 & 182 \\
\hline Median & 68 & - & 13 & 0 & 29 & 181 \\
\hline
\end{tabular}

Abbreviations: F, female; FU, follow-up; M, male; OS, overall survival; Post-PV MF, post-polycythemic myelofibrosis.

the post-polycythemic myelofibrosis cases with leukocytosis (Table 4). Control cases had been treated during the polycythemic phase similarly to the cases group, using phlebotomies and various combinations of hydroxyurea, anagrelide, and aspirin; at time of post-polycythemic myelofibrosis diagnosis phlebotomies had been suspended in all cases. Compared with the neutrophilic cases, control cases showed lower WBC and neutrophil counts $(P=0.001$ and 0.007 , respectively) and also a lower absolute monocyte count. However, when comparing the percentage of monocytes, no difference was noted. All other features were similar in the two groups.

\section{Morphologic and Immunohistochemical Analysis of Bone Marrow}

Marrow fibrosis was measured as grade 2 in 7 cases $(70 \%)$ of post-polycythemic myelofibrosis with leukocytosis and as grade 3 in the remaining 3 $(30 \%)$. Cellularity was increased for the age in all cases (mean: 95\%). Megakaryocytes showed marked polymorphism ranging from cells displaying normally folded nuclei to hyperlobulated and/or abnormally lobulated ones. Tight clusters of megakaryocytes were only rarely observed. None of the cases showed an increased number of blasts, with CD34+ cells ranging between 1 and $2 \%$ in all. Five marrow biopsies $(50 \%)$ displayed a markedly increased myeloid proliferation with preponderance of mature neutrophilic forms (Figure 1), which was reflected by the increased myeloid:erythroid ratio ranging between $6: 1$ and 17:1 observed in the corresponding bone marrow aspirate smear; the remaining five marrows showed a normal or only mildly increased myeloid:erythroid ratio, ranging from 1 to 5:1. For case 10, two sequential biopsies taken during post-polycythemic myelofibrotic stage before and after the development of leukocytosis were available for review. In the second one the myeloid:erythroid ratio was markedly increased compared with the first biopsy (6:1 vs. 1:2, respectively). However, no other changes were observed, including degree of marrow fibrosis (grade 2) and the percentage of CD34+ blasts $(1 \%)$. In case 2 , the spleen was removed a few months after the diagnosis of post-polycythemic myelofibrosis and the onset of leukocytosis: morphologic analysis showed diffuse extramedullary hematopoiesis with preponderance of neutrophilic granulocytes stuffing the red pulp.

The morphologic findings seen in the control group are found summarized in Table 4. In the comparison between the control and the neutrophilic group the proportion of marrows with grade 3 fibrosis was slightly higher and the mean myeloid:erythroid ratio was lower among controls $(P=0.02$ for both), whereas other morphologic features were similar.

\section{Peripheral Blood Smear}

Peripheral blood smears showed mostly cytologically unremarkable neutrophils in all cases of postpolycythemic myelofibrosis with leukocytosis (Figure 1). Significant nuclear hypersegmentation was observed only in one case (case 3). Immature myeloid elements accounted for $\geq 10 \%$ of peripheral blood white cells in four patients (cases 2, 3, 4, and 7, Table 2). In three (cases 2, 3, and 4) this feature was associated with the presence of marked myeloid proliferation in the bone marrow and an elevated myeloid:erythroid ratio, whereas in case 7 the myeloid:erythroid ratio was only borderline elevated. Cases with $<10 \%$ of circulating immature myeloid elements showed a normal myeloid:erythroid ratio, except for case 9. All cases, including those cases with higher percentages of circulating immature elements, showed $\leq 2 \%$ blasts in both marrow and peripheral blood (Table 2).

In the control group the percentage of circulating immature myeloid elements was comparable to that 
Table 2 Summary of the main morphologic features of post-polycythemic myelofibrosis biopsies and of WBC and neutrophil counts at time of biopsy. Karyotype and molecular status of JAK2 are also reported

\begin{tabular}{|c|c|c|c|c|c|c|c|c|c|c|c|c|}
\hline Case & $\begin{array}{l}B M \\
\text { fibrosis } \\
\text { grade }\end{array}$ & $M / E$ ratio & $\begin{array}{l}\text { CD34+ } \\
\text { blasts in } \\
\text { BM }\end{array}$ & $H b \operatorname{gr} / d L$ & $\begin{array}{c}W B C \\
\times 10^{9} / 1\end{array}$ & $\begin{array}{l}\text { Neutrophils } \\
\times 10^{9} / 1(\%)\end{array}$ & $\begin{array}{l}\text { Monocytes } \\
\times 10^{9} / I(\%)\end{array}$ & $\begin{array}{l}\text { Platelets } \\
\times 10^{9} / 1\end{array}$ & $\begin{array}{c}\text { Immature } \\
\text { myeloid } \\
\text { cells in } \\
P B \%\end{array}$ & $\begin{array}{l}\text { Blasts \% } \\
\text { in } P B\end{array}$ & Karyotype & JAK2 \\
\hline 1 & 2 & $3 / 1$ & $0 \%$ & 14.4 & 17.5 & $13.1(75)$ & $0.5(3)$ & 595 & $<1 \%$ & $1 \%$ & $46, X X$ & Mutated \\
\hline 2 & 3 & $17 / 1$ & $2 \%$ & 10.4 & 81.3 & $50.4(62)$ & $4.0(5)$ & 217 & $14 \%$ & $2 \%$ & $\begin{array}{c}\text { 46,XX,del(13)(q12q14)[14]/ } \\
\text { 46,idem,+add(1)(p11), - 6[5]/ } \\
\text { 46,idem,del(4)(q21)[1] }\end{array}$ & Mutated \\
\hline 3 & 3 & $9 / 1$ & $0 \%$ & 12.4 & 89.7 & $70.9(79)$ & $1.8(2)$ & 223 & $15 \%$ & $1 \%$ & $46, X Y$ & Mutated \\
\hline 4 & 3 & $9 / 1$ & $1 \%$ & 10.2 & 22.2 & $18.6(84)$ & $1.1(5)$ & 572 & $10 \%$ & $2 \%$ & na & Mutated \\
\hline 5 & 2 & $1 / 1$ & $1 \%$ & 13.6 & 19.7 & $16.5(84)$ & $0.8(4)$ & 352 & $<1 \%$ & $2 \%$ & $48, X Y,+8,+9[2] / 46 X Y[17]$ & Mutated \\
\hline 6 & 2 & $3 / 1$ & $2 \%$ & 14.1 & 18 & 15 (83) & $0.5(3)$ & 562 & $4 \%$ & $0 \%$ & na & Mutated \\
\hline 7 & 2 & $5 / 1$ & $1 \%$ & 13.9 & 72.6 & $60.8(84)$ & $2.2(3)$ & 267 & $16 \%$ & $0 \%$ & $46, X Y$ & Mutated \\
\hline 8 & 2 & $3 / 1$ & $1 \%$ & 14.2 & 16.1 & $14.1(87)$ & $0.6(4)$ & 403 & $5 \%$ & $0 \%$ & $46, X Y$ & Mutated \\
\hline 9 & 2 & $8 / 1$ & $2 \%$ & 13 & 27.9 & $25.7(92)$ & $0.8(3)$ & 110 & $<1 \%$ & $0 \%$ & $46, X Y$ & Mutated \\
\hline 10 & 2 & $6 / 1$ & $1 \%$ & 11.2 & 43 & $35.5(83)$ & $1.3(3)$ & 1434 & $<1 \%$ & $1 \%$ & $\begin{array}{c}46, \mathrm{XY},+1, \operatorname{der}(1 ; 15)(q 10 ; q 10) \\
{[13] / 46, \mathrm{XY}[16]}\end{array}$ & Mutated \\
\hline Range & & $1-17 / 1$ & $0-2 \%$ & $8.5-14.4$ & $16.1-89.7$ & $13.1-70.9(62-92)$ & $0.5-4.0(2-5)$ & 110-1434 & $<1-16 \%$ & $0-2 \%$ & & \\
\hline Mean & & $6.4 / 1$ & $1.1 \%$ & 12.7 & 40.8 & $32.1(81)$ & $1.4(4)$ & 473.5 & $7 \%$ & $0.9 \%$ & & \\
\hline Median & & $5.5 / 1$ & $1 \%$ & 13.3 & 25.1 & $22.2(84)$ & $1(3)$ & 377.5 & $5 \%$ & $1 \%$ & & \\
\hline
\end{tabular}

Abbreviations: $\mathrm{BM}$, bone marrow; $\mathrm{Hb}$, hemoglobin; M/E ratio, myeloid:erythroid ratio; na, not available; PB, peripheral blood; WBC, white blood cell.

observed in the neutrophilic group $(9 \%$ vs $7 \%$, respectively; Table 4): higher values were observed in cases with grade 3 marrow fibrosis. Blast counts were not increased in any of the control cases.

\section{Cytogenetic Analysis}

Cytogenetic results were available in 8 out of 10 patients with post-polycythemic myelofibrosis and leukocytosis. Five cases showed a normal karyotype $(62 \%)$ whereas three $(38 \%)$ displayed chromosomal abnormalities. Only case 2 showed a complex karyotype, ie, with $\geq 3$ alterations, and multiple clones (Table 2). Cytogenetic results were available for 18 of the control cases: 6 (33\%) showed a normal karyotype, whereas 12 (66\%) showed alterations with 5 complex karyotypes. Abnormal karyotypes were more frequent in the control group, although only a borderline statistical significance was reached $(P=0.08)$. All cases in both groups were negative for $t(9 ; 22)$ and $B C R-A B L 1$ and there was no cytogenetic evolution or change in $B C R-A B L 1$ status associated with the development of leukocytosis.

\section{Molecular Analysis}

All cases, both in the neutrophilic cases and in the control group, were positive for JAK2V617F mutation. All cases of post-polycythemic myelofibrosis with leukocytosis showed wild-type alleles for CSF3R, SETBP1, and of SRSF2.

\section{Survival Analysis}

Four $(40 \%)$ of the neutrophilic post-polycythemic myelofibrosis patients (cases 2, 4, 7, and 8) died at a median interval of 11 months after the onset of leukocytosis, three $(30 \%)$ of causes related to the progression of polycythemia vera and one from unrelated causes (case 8). The latter was excluded from the survival analysis. An additional patient (case 3) showed worsening disease and became transfusion dependent (Table 3). Among the 23 controls only $2(9 \%)$ died, both from progression of disease to blastic phase. Survival analysis showed a significantly shorter overall survival from time of polycythemia vera diagnosis in patients with leukocytosis compared with controls (log-rank test: $P=0.046$; Figure 2). When comparing survival from the time of post-polycythemic myelofibrosis diagnosis, no difference was noted between the two groups.

\section{Discussion}

Prognostic evaluation of polycythemia vera is primarily based on clinical features and blood count results obtained at the time of diagnosis. Two types of disease progression are currently recognized for polycythemia vera in the WHO 2008 classification: a late stage dominated by marrow fibrosis and hematopoietic failure, termed post-polycythemic myelofibrosis and leukemic transformation usually separated into accelerated and blast phases. ${ }^{1}$ Fibrotic evolution and blast/accelerated phase are forms of progression that polycythemia vera shares with other myeloproliferative neoplasms. However, other rarer types of progression may also be observed in myeloproliferative neoplasms. Our group has recently reported that some patients with primary myelofibrosis may undergo a form of disease acceleration characterized by the development of sustained absolute monocytosis with marrow changes resembling those seen in chronic myelomonocytic leukemia. ${ }^{8,9}$

In this study we describe for the first time a group of polycythemia vera patients that developed persistent significant leukocytosis around the time or after their evolution to post-polycythemic myelofibrosis 
Table 3 Summary of treatment regimens, prior and following the development of leukocytosis, and of clinical status of patients

\begin{tabular}{|c|c|c|c|c|c|}
\hline \multirow[b]{2}{*}{ Case } & \multicolumn{2}{|c|}{ Treatment } & \multirow[b]{2}{*}{$\begin{array}{c}\text { Thrombotic/ } \\
\text { hemorrhagic events }\end{array}$} & Clinical Status & \multirow[b]{2}{*}{ Cause of death } \\
\hline & Polycythemic phase & Post-PV MF & & Post-neutrophilia & \\
\hline 1 & Phlebotomies, hydroxyurea & Aspirin, hydroxyurea & na & Alive & \\
\hline 2 & $\begin{array}{l}\text { Phlebotomies, hydroxyurea, } \\
\text { anagrelide }\end{array}$ & Interferon & na & Dead & $\begin{array}{l}\text { Worsening } \\
\text { cytopenias }\end{array}$ \\
\hline 3 & na & Aspirin, azacitidine & $\begin{array}{l}\text { Portal and splenic } \\
\text { vein thrombosis }\end{array}$ & $\begin{array}{l}\text { Worsening anemia and } \\
\text { thrombocytopenia with } \\
\text { circulating blasts }\end{array}$ & \\
\hline 4 & Phlebotomies & $\begin{array}{l}\text { Hydroxyurea, } \\
\text { anagrelide }\end{array}$ & na & Dead & $\begin{array}{l}\text { Hemorrhagic shock } \\
\text { due to GI bleeding }\end{array}$ \\
\hline 5 & Phlebotomies, aspirin, hydroxyurea & Aspirin, hydroxyurea & 0 & Alive & \\
\hline 6 & $\begin{array}{l}\text { Phlebotomies, aspirin, hydroxyurea, } \\
\text { interferon, melphalan }\end{array}$ & Aspirin, hydroxyurea & 0 & Lost at follow-up & \\
\hline 7 & Phlebotomies, aspirin, hydroxyurea & aspirin & 1 TIA & Dead & AML \\
\hline 8 & Phlebotomies, aspirin, hydroxyurea & Aspirin, hydroxyurea & DVT & Dead & $\begin{array}{l}\text { Progression of SCC } \\
\text { of penis }\end{array}$ \\
\hline 9 & Phlebotomies, aspirin & Aspirin, hydroxyurea & 0 & Alive & \\
\hline 10 & Phlebotomies, aspirin, hydroxyurea & $\begin{array}{l}\text { Aspirin, hydroxyurea, } \\
\text { ruxolitinib }\end{array}$ & 0 & Alive & \\
\hline
\end{tabular}

Abbreviations: AML, acute myeloid leukemia; DVT, deep vein thrombosis; GI, gastrointestinal; na, not available; Post-PV MF, post-polycythemic myelofibrosis; SCC, squamous cell carcinoma; TIA, transient ischemic attack.

Table 4 Comparison of the clinical and morphological features of the patients in the control group and the post-polycythemic myelofibrosis patients with leukocytosis. Mean values are reported, unless otherwise specified

\begin{tabular}{|c|c|c|c|}
\hline & Control group $(\mathrm{n}=23)$ & Post-PV MF with leukocytosis $(\mathrm{n}=10)$ & $\mathrm{P}$-value \\
\hline Age (range) & $65(45-79)$ & $66(39-75)$ & NS \\
\hline $\operatorname{Sex}(M / F)$ & $16 / 7$ & $7 / 3$ & NS \\
\hline Hemoglobin (g/l) & 11.8 & 12.7 & NS \\
\hline WBC $\times 10^{9} / 1$ & 14.4 & 40.8 & 0.001 \\
\hline Neutrophils $\times 10^{9} / 1(\%)$ & $11.5(72)$ & $32.1(81)$ & $0.007(0.06)$ \\
\hline Monocytes × $10^{9} / 1(\%)$ & $0.4(4.2)$ & $1.4(4)$ & 0.01 (NS) \\
\hline Platelets $\times 10^{9} / 1$ & 280.1 & 473.5 & NS \\
\hline Splenomegaly (yes/no) & $17 / 2$ & $7 / 3$ & - \\
\hline Cytogenetics (normal/altered) & $6 / 12$ & $5 / 3$ & NS \\
\hline JAK2V617 (mutated/WT) & $23 / 0$ & $10 / 0$ & NS \\
\hline Cellularity (\%) & 90 & 95 & NS \\
\hline Marrow fibrosis: grade 2/grade 3 (ratio) & $8 / 15(0.5)$ & 7/2 (3.5) & 0.02 \\
\hline Myeloid:erythroid ratio & 6.4 & 3.9 & 0.02 \\
\hline CD34+ blasts in marrow (\%) & $<1$ & 1.1 & NS \\
\hline Circulating immature myeloid elements (\%) & 9 & 7 & NS \\
\hline Circulating blasts (\%) & 1 & $<1$ & NS \\
\hline Median overall survival (months) & 252 & 181 & - \\
\hline Median FU after Post-PV MF diagnosis (months) & 38 & 37 & - \\
\hline Mortality due to disease progression & $9 \%$ & $30 \%$ & - \\
\hline
\end{tabular}

Abbreviations: F, female; FU, follow-up; M, male; NS, not significant; Post-PV MF, post-polycythemic myelofibrosis; WBC, white blood cells; WT, wild type.

and show that this occurrence is associated with a worse overall survival. There is previously published information dealing with leukocytosis and neutrophilia in patients with polycythemia vera. Tefferi et al. ${ }^{11}$ and Bonicelli et al. ${ }^{10}$ have recently identified leukocytosis (WBC: $>13$ or $\geq 15 \times 10^{9} / 1$, respectively) occurring in polycythemia vera at diagnosis or during the polycythemic phase as an independent adverse prognostic factor, confirming early preliminary observations. ${ }^{17,18}$ However, all these previous studies were focused exclusively on patients in polycythemic phase (not post-polycythemic) and lacked detailed WBC differential counts and/or a description of the observed bone marrow findings. In the present study we adopted the threshold of $\geq 13 \times 10^{9} / \mathrm{l}$ WBC to define significant leukocytosis because this cut-off is commonly used in the WHO 2008 monograph to define leukocytosis in the setting of different myeloid entities. ${ }^{19-22}$

Marked myeloid proliferation and markedly increased myeloid:erythroid ratio are not features commonly observed in polycythemia vera marrows, as highlighted by the comparison of neutrophilic cases with the cases of the control group. The appearance of a 'myeloid shift' in marrow morphology seems to be strictly associated with the onset of 

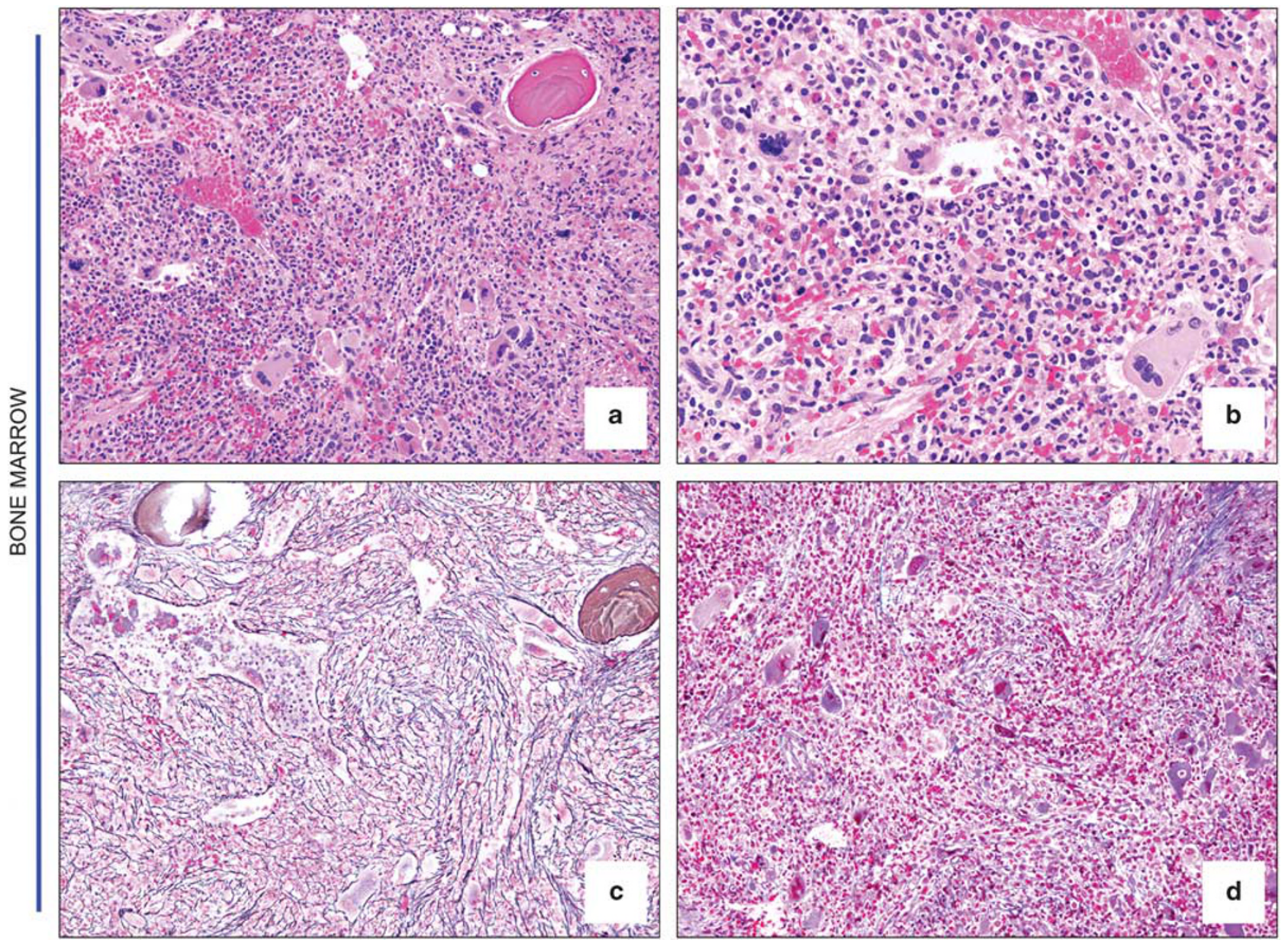

Figure 1 Bone marrow biopsy of a case of post-polycythemic myelofibrosis with leukocytosis (case 2). (a and b): (a, $\times 10$ magnification, $\mathbf{b}$, $\times 20$ ) hypercellular bone marrow with a marked neutrophilic proliferation. Note the presence of pleomorphic megakaryocytes consistent with polycythemia vera. The megakaryocytes' morphology seen in the neutrophilic cases of post-polycythemic myelofibrosis was not different from that observed in the cases included in the control group. (c and $\mathbf{d})$ : reticulin $(\mathbf{c}, \times 10)$ and trichrome $(\mathbf{d}, \times 10)$ stains showing grade 3 fibrosis that confirmed the diagnosis of post-polycythemic myelofibrosis. (e and $\mathbf{f}):(\mathbf{e}, \times 40 ; \mathbf{f}, \times 60)$ peripheral blood smear showing markedly increased WBC with neutrophilia and increased number of circulating immature myeloid precursors (WBC: $81.3 \times 109 / 1,62 \%$ neutrophils). ( $\mathrm{g}$ and $\mathbf{h}):(\mathrm{g}, \times 20 ; \mathbf{h}, \times 20)$ spleen removed a few months after the onset of leukocytosis showing extramedullary hematopoiesis dominated by a neutrophil-rich granulocytic proliferation (g). The granulocytic cells are highlighted by their positivity for myeloperoxidase (anti-MPO immunohistochemistry) (h). WBC, white blood cells.

peripheral neutrophilia: the comparison of bone marrow biopsies taken before and after the onset of neutrophilic leukocytosis and also the morphologic examination of a spleen removed during the myelofibrotic phase showed that both marrow and extramedullary hematopoiesis were dominated by a granulocytic proliferation composed of segmented neutrophils. However, a marked myeloid shift was not evident in all cases.

The findings observed in these cases of postpolycythemic myelofibrosis with leukocytosis and neutrophilia can pose diagnostic difficulties. From a morphological point of view, some of these case may in fact superficially resemble chronic neutrophilic leukemia, a rare subtype of $B C R-A B L 1$ negative myeloproliferative neoplasm ${ }^{20}$ characterized by the prevalence of normally segmented, non-dysplastic neutrophils. In five of our post-polycythemic myelofibrosis cases with neutrophilia, similarly to chronic neutrophilic leukemia, the bone marrow biopsies showed a striking myeloid proliferation with abundance of neutrophils, high myeloid:erythroid ratios, no increase in the number of blasts and, again, no evidence of significant dysplasia. In contrast, in other four cases the presence of $\geq 10 \%$ immature myeloid cells in peripheral blood in addition to the presence of a WBC $\geq 13 \times 10^{9} / 1$ may suggest a diagnosis of atypical chronic myeloid leukemia $B C R-A B L 1$ negative, a rare myelodysplastic/myeloproliferative neoplasm, but the absence of dysplastic features in our cases tended to exclude such possibility. Although we observed an increase in the absolute counts of monocytes in the case group compared with the control group, the percentage of monocytes never exceeded $5 \%$. Thus, the increase in monocyte count seen in cases of post-polycythemic myelofibrosis with neutrophilia most likely represents a minor consequence of the high WBC. 

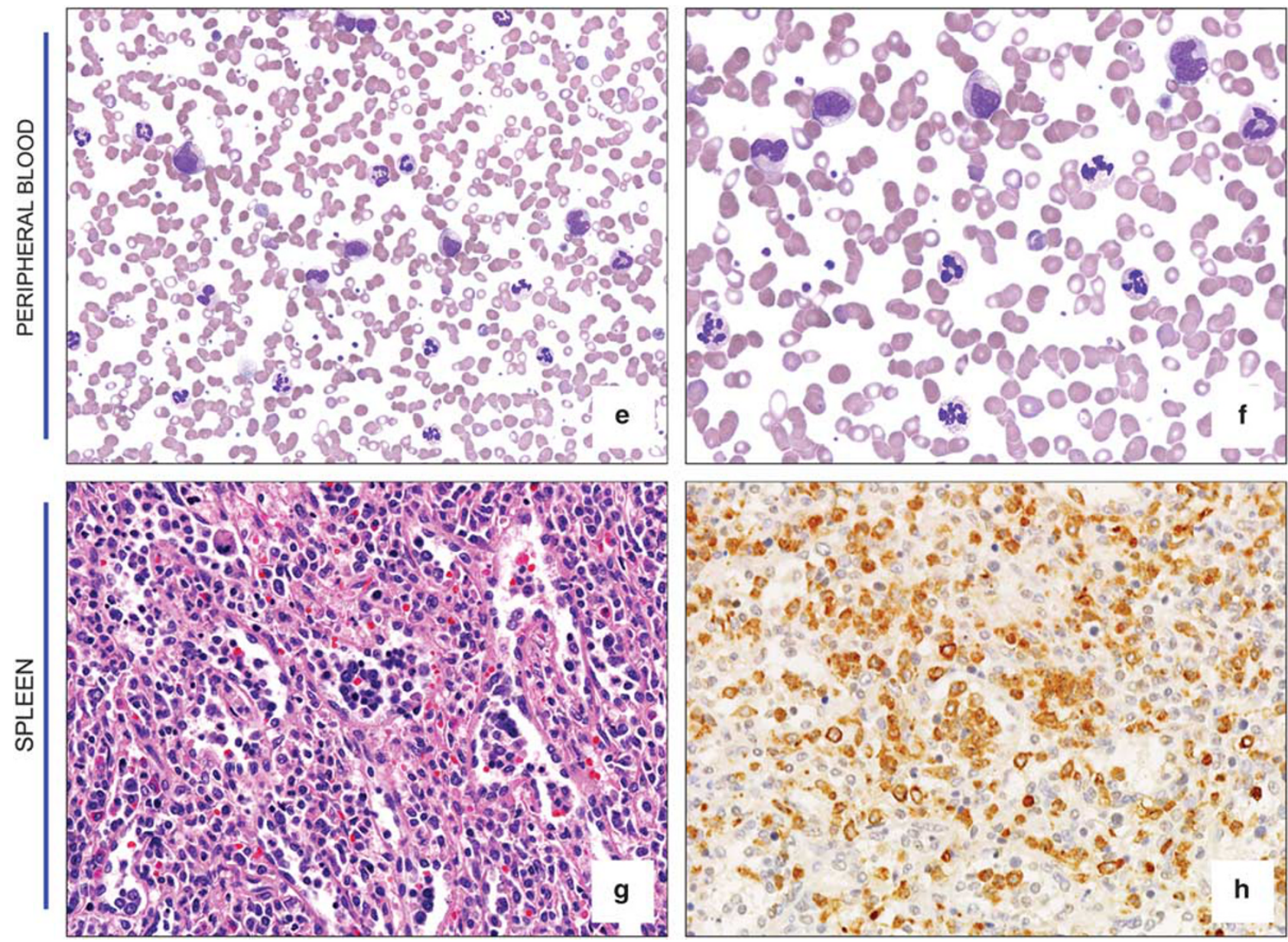

Figure 1 Continued.

The concurrence of elevated counts of peripheral neutrophils and pronounced mature myeloid proliferation in marrow prompted the hypothesis that at least some cases could share a biological relationship with the other types of myeloproliferative neoplasms that typically show increased neutrophilic and/or myeloid proliferation, as chronic neutrophilic leukemia, atypical chronic myeloid leukemia $B C R-A B L 1$ negative or chronic myelomonocytic leukemia. All cases retained their $J A K 2 \mathrm{~V} 617 \mathrm{~F}$ mutation positivity and remained negative for $B C R-A B L 1$ fusion. To explore other possible mechanisms involved with the development of leukocytosis, we investigated eight cases for mutations of CSF3R, SETBP1, and SRSF2 genes. Truncation mutations of the receptor cytoplasmic domain for $C S F 3 R$ have been recently identified as a highly sensitive and specific molecular marker for $\mathrm{CNL}^{23-25}$ and SETBP1 is frequently mutated in atypical chronic myeloid leukemia ${ }^{26}$ in other myelodysplastic syndromes, myelodysplastic/myeloproliferative neoplasms ${ }^{27}$ or chronic myelomonocytic leukemia. ${ }^{28}$ SRSF2 mutations has been described in $47 \%$ of chronic myelomonocytic leukemia ${ }^{29}$ and, although less frequently, in other types of myelodysplastic and myeloproliferative neoplasms. ${ }^{30-32}$ All neutrophilic post-polycythemic myelofibrosis cases showed only wild-type alleles for these genes, a finding that suggests that the neutrophilic proliferation arising during the fibrotic stages of polycythemia vera is caused by mechanisms biologically distinct from those involved in chronic neutrophilic leukemia or atypical chronic myeloid leukemia.

Cytogenetic abnormalities and complex karyotypes are a common feature of post-polycythemic myelofibrosis, ${ }^{6}$ and are considered evidence of progressive accumulation of genetic damage. However, among the neutrophilic cases an abnormal karyotype was found in $38 \%$ of cases, fewer than in the control group. The presence of chromosomal abnormalities did not correlate with the degree of peripheral neutrophilia or neutrophilic proliferation in marrow. In fact, among the four cases showing the highest myeloid:erythroid ratios and neutrophil counts cytogenetic results were mixed: two patients showed chromosomal anomalies but the remaining two displayed normal karyotypes. Moreover, there was no association between the development of leukocytosis and any specific alteration.

In spite of these negative results, the most likely explanation is that a yet undiscovered molecular 


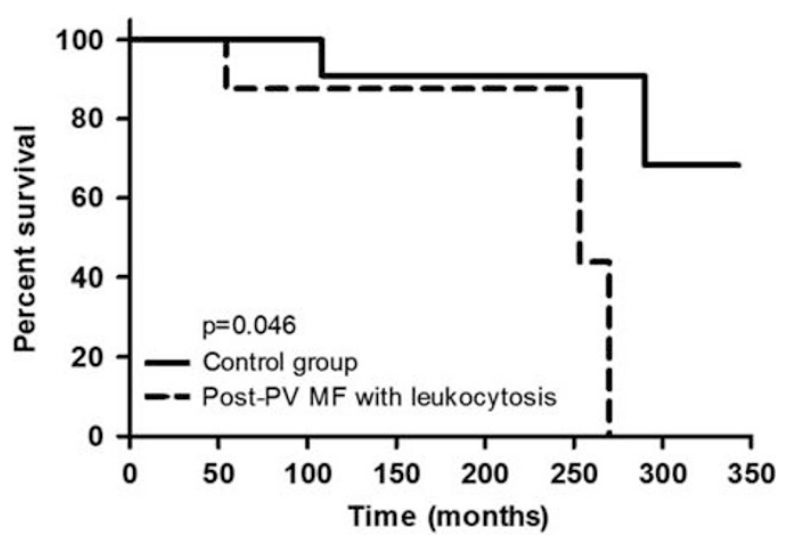

Figure 2 Kaplan-Meier curves describing overall survival from the time of diagnosis of polycythemia vera in the case and in the control groups. Overall survival resulted significantly shorter (logrank test: $P=0.046$ ) in cases of post-polycythemic myelofibrosis with leukocytosis than in the control group.

alteration such as the presence of a myeloid cell subclone present at disease onset or acquired later in the course of polycythemia vera, succeeds in becoming dominant during the late stage of the disease. The presence of such clonal instability might account both for the observed phenotypic shift and the worse overall survival of these patients. Clonal complexity as a mechanism underlying phenotypic shifts in myeloid neoplasm has been recently reviewed by Itzykson et al. ${ }^{33}$ The more widely known and best characterized example includes the development in cases of JAK2 mutation-positive myeloproliferative neoplasm of two different leukemic clones, one with $J A K 2$ mutation-positive blasts and one with JAK2 wild-type blasts; another example of this mechanism could explain the selection of JAK2 positive/ $B C R-A B L 1$ negative clones from those rare cases of myeloproliferative neoplasms with concurrent $B C R-A B L 1$ and JAK2 mutation-positivity treated with imatinib. ${ }^{34-36}$ Therefore, a more comprehensive mutational analysis with more numerous samples might be able to better estimate the type and frequency of molecular alterations associated with this uncommon event.

Our data suggest potentially important clinical implications. Among neutrophilic patients three died soon after the onset of leukocytosis for disease progression and an additional patient showed signs of progressively worsening disease. Thus, the death rate in the neutrophilic post-polycythemic myelofibrosis group was higher than in the control group. This observation was supported by survival analysis that showed shorter overall survival, calculated from the time of diagnosis of polycythemia vera, in the cohort of patients who went on to develop neutrophilia than in the control group. However, when survival was calculated from the time of progression to post-polycythemic myelofibrosis no difference was evident between the two groups. This latter finding might be explained by the limited number of patients included in the study and/or by the fact that myelofibrotic progression of polycythemia vera carries already an inherently very poor prognosis irrespectively of additional adverse findings such as the late development of leukocytosis or a higher frequency of cytogenetic alterations. ${ }^{6}$

In summary, the occurrence of neutrophilic progression in advanced stage polycythemia vera patients should be recognized because of its potential adverse impact. A better understanding of the pathogenic mechanisms underlying this phenomenon may help the development of newer targeted treatments aimed at preventing disease progression in polycythemia vera patients.

\section{Acknowledgments}

Leonardo Boiocchi was supported by a grant of Associazione Davide Rodella onlus, Montichiari (BS), Italy.

\section{Disclosure/conflict of interest}

The authors declare no conflict of interest.

\section{References}

1 Thiele J, Kvasnicka HM, Orazi A et al. Polycythaemia veraIn:Swerdlow SH, Campo E, Harris NL et al. (eds) WHO Classification of Tumours of Haematopoietic and Lymphoid Tissues. IARC: Lyon, 2008, pp 40-43.

2 Thiele J, Kvasnicka HM, Orazi A. Bone marrow histopathology in myeloproliferative disorders-current diagnostic approach. Semin Hematol 2005;42:184-195.

3 Silver RT, Chow W, Orazi A et al. Evaluation of WHO criteria for diagnosis of polycythemia vera: a prospective analysis. Blood 2013;122:1881-1886.

4 Pozdnyakova O, Hasserjian RP, Verstovsek S et al. Impact of bone marrow pathology on the clinical management of Philadelphia chromosome-negative myeloproliferative neoplasms. Clin Lymphoma Myeloma Leuk 2015;15:253-261.

5 Barosi G, Mesa RA, Thiele J et al. Proposed criteria for the diagnosis of post-polycythemia vera and postessential thrombocythemia myelofibrosis: a consensus statement from the International Working Group for Myelofibrosis Research and Treatment. Leukemia 2008; 22:437-438.

6 Boiocchi L, Mathew S, Gianelli U et al. Morphologic and cytogenetic differences between post-polycythemic myelofibrosis and primary myelofibrosis in fibrotic stage. Mod Pathol 2013;26:1577-1585.

7 Boiocchi L, Espinal-Witter R, Geyer JT et al. Development of monocytosis in patients with primary myelofibrosis indicates an accelerated phase of the disease. Mod Pathol 2013;26:204-212.

8 Orazi A, Germing U. The myelodysplastic/myeloproliferative neoplasms: myeloproliferative diseases with dysplastic features. Leukemia 2008;22:1308-1319.

9 Orazi A, Bennet JM, Germing U et al. Chronic myelomonocytic leukemiaIn:Swerdlow SH, Campo E, Harris NL et al. (eds) WHO Classification of Tumours of 
Haematopoietic and Lymphoid Tissues. IARC: Lyon, 2008, pp 76-79.

10 Bonicelli G, Abdulkarim K, Mounier M et al. Leucocytosis and thrombosis at diagnosis are associated with poor survival in polycythaemia vera: a populationbased study of 327 patients. Br J Haematol 2013;160: 251-254.

11 Tefferi A, Rumi E, Finazzi G et al. Survival and prognosis among 1545 patients with contemporary polycythemia vera: an international study. Leukemia 2013;27:1874-1881.

12 Barbui T, Carobbio A, Rambaldi A et al. Perspectives on thrombosis in essential thrombocythemia and polycythemia vera: is leukocytosis a causative factor? Blood 2009;114:759-763.

13 Falanga A, Marchetti M, Barbui T et al. Pathogenesis of thrombosis in essential thrombocythemia and polycythemia vera: the role of neutrophils. Semin Hematol 2005;42:239-247.

14 Thiele J, Kvasnicka HM, Facchetti F et al. European consensus on grading bone marrow fibrosis and assessment of cellularity. Haematologica 2005;90:1128-1132.

15 Kui JS, Espinal-Witter R, Wang YL. Laboratory detection of JAK2V617F in human myeloproliferative neoplasms. Methods Mol Biol 2013;999:41-57.

16 Federmann B, Abele M, Rosero Cuesta DS et al. The detection of SRSF2 mutations in routinely processed bone marrow biopsies is useful in the diagnosis of chronic myelomonocytic leukemia. Hum Pathol 2014;45:2471-2479.

17 Passamonti F, Rumi E, Pungolino E et al. Life expectancy and prognostic factors for survival in patients with polycythemia vera and essential thrombocythemia. Am J Med 2004;117:755-761.

18 Gangat N, Strand J, Li CY et al. Leucocytosis in polycythaemia vera predicts both inferior survival and leukaemic transformation. Br J Haematol 2007;138: 354-358.

19 Baumann I, Bennett JM, Niemeyer CM et al. Juvenile Myelomonocytic Leukaemia, In: WHO Classification of Tumours of Haematopoietic and Lymphoid Tissues. IARC: Lyon, 2008, pp 82-84.

20 Vardiman JW, Melo JV, Baccarani M et al. Chronic myelogenous leukemia, $B C R-A B L 1$ positive. In: Swerdlow SH, Campo E, Harris NL et al. (eds) WHO Classification of Tumours of Haematopoietic and Lymphoid Tissues. IARC: Lyon, 2008, pp 32-37.

21 Vardiman JW, Bennett JM, Bain BJ et al. Atypical Chronic Myeloid Leukemia, BCR-ABL1 Negative, In: WHO Classification of Tumours of Haematopoietic and Lymphoid Tissues. IARC: Lyon, 2008, pp 80-81.

22 Vardiman JW, Bennett JM, Bain BJ et al. Myelodysplastic/Myeloproliferative Neoplasm, Unclassifiable, In: WHO Classification of Tumours of Haematopoietic and Lymphoid Tissues. IARC: Lyon, 2008, pp 85-86.
23 Pardanani A, Lasho TL, Laborde RR et al. CSF3R T618I is a highly prevalent and specific mutation in chronic neutrophilic leukemia. Leukemia 2013;27:1870-1873.

24 Maxson JE, Gotlib J, Pollyea DA et al. Oncogenic CSF3R mutations in chronic neutrophilic leukemia and atypical CML. N Engl J Med 2013;368:1781-1790.

25 Elliott MA, Tefferi A. The molecular genetics of chronic neutrophilic leukaemia: defining a new era in diagnosis and therapy. Curr Opin Hematol 2014;21:148-154.

26 Piazza R, Valletta S, Winkelmann N et al. Recurrent SETBP1 mutations in atypical chronic myeloid leukemia. Nat Genet 2013;45:18-24.

27 Fernandez-Mercado M, Pellagatti A, Di Genua C et al. Mutations in SETBP1 are recurrent in myelodysplastic syndromes and often coexist with cytogenetic markers associated with disease progression. Br J Haematol 2013;163:235-239.

28 Laborde RR, Patnaik MM, Lasho TL et al. SETBP1 mutations in 415 patients with primary myelofibrosis or chronic myelomonocytic leukemia: independent prognostic impact in CMML. Leukemia 2013;27: 2100-2102.

29 Meggendorfer M, Roller A, Haferlach T et al. SRSF2 mutations in 275 cases with chronic myelomonocytic leukemia (CMML). Blood 2012;120:3080-3088.

30 Hanssens K, Brenet F, Agopian J et al. SRSF2-p95 hotspot mutation is highly associated with advanced forms of mastocytosis and mutations in epigenetic regulator genes. Haematologica 2014;99:830-835.

31 Zhang SJ, Rampal R, Manshouri $\mathrm{T}$ et al. Genetic analysis of patients with leukemic transformation of myeloproliferative neoplasms shows recurrent SRSF2 mutations that are associated with adverse outcome. Blood 2012;119:4480-4485.

$32 \mathrm{Wu}$ SJ, Kuo YY, Hou HA et al. The clinical implication of SRSF2 mutation in patients with myelodysplastic syndrome and its stability during disease evolution. Blood 2012;120:3106-3111.

33 Itzykson R, Kosmider O, Renneville A et al. Clonal architecture of chronic myelomonocytic leukemias. Blood 2013;121:2186-2198.

34 Beer PA, Delhommeau F, LeCouedic JP et al. Two routes to leukemic transformation after a JAK2 mutation-positive myeloproliferative neoplasm. Blood 2010;115:2891-2900.

35 Hussein K, Bock O, Seegers A et al. Myelofibrosis evolving during imatinib treatment of a chronic myeloproliferative disease with coexisting BCR-ABL translocation and JAK2V617F mutation. Blood 2007;109:4106-4107.

36 Hussein K, Bock O, Theophile $\mathrm{K}$ et al. Biclonal expansion and heterogeneous lineage involvement in a case of chronic myeloproliferative disease with concurrent MPLW515L/JAK2V617F mutation. Blood 2009;113:1391-1392. 